\title{
Frequent Loss of Genome Gap Region in 4p16.3 Subtelomere in Early-Onset Type 2 Diabetes Mellitus
}

\author{
Hirohito Kudo, ${ }^{1}$ Mitsuru Emi, ${ }^{2}$ Yasushi Ishigaki, ${ }^{1}$ Uiko Tsunoda, ${ }^{1}$ Yoshinori Hinokio, ${ }^{1}$ \\ Miho Ishii, ${ }^{2}$ Hidenori Sato, ${ }^{2}$ Tetsuya Yamada, ${ }^{3}$ Hideki Katagiri, ${ }^{3}$ and Yoshitomo Oka ${ }^{1}$ \\ ${ }^{1}$ Division of Molecular Metabolism and Diabetes, Tohoku University Graduate School of Medicine, 2-1 Seiryo-machi, Aoba-ku, \\ Sendai, Miyagi 980-8575, Japan \\ ${ }^{2}$ CNV Laboratory, DNA Chip Research Institute, 1-1-43 Suehiro-cho, Tsurumi-ku Yokohama, Kanagawa 230-0045, Japan \\ ${ }^{3}$ Department of Metabolic Diseases, Center for Metabolic Diseases, Tohoku University Graduate School of Medicine, \\ 2-1 Seiryo-machi, Aoba-ku, Sendai, Miyagi 980-8575, Japan
}

Correspondence should be addressed to Hideki Katagiri, katagiri@med.tohoku.ac.jp

Received 6 October 2010; Revised 21 February 2011; Accepted 30 March 2011

Academic Editor: Kazuya Yamagata

Copyright (c) 2011 Hirohito Kudo et al. This is an open access article distributed under the Creative Commons Attribution License, which permits unrestricted use, distribution, and reproduction in any medium, provided the original work is properly cited.

A small portion of Type 2 diabetes mellitus (T2DM) is familial, but the majority occurs as sporadic disease. Although causative genes are found in some rare forms, the genetic basis for sporadic T2DM is largely unknown. We searched for a copy number abnormality in 100 early-onset Japanese T2DM patients (onset age $<35$ years) by whole-genome screening with a copy number variation BeadChip. Within the 1.3-Mb subtelomeric region on chromosome $4 \mathrm{p} 16.3$, we found copy number losses in early-onset T2DM (13 of 100 T2DM versus one of 100 controls). This region surrounds a genome gap, which is rich in multiple low copy repeats. Subsequent region-targeted high-density custom-made oligonucleotide microarray experiments verified the copy number losses and delineated structural changes in the 1.3-Mb region. The results suggested that copy number losses of the genes in the deleted region around the genome gap in 4p16.3 may play significant roles in the etiology of T2DM.

\section{Introduction}

Type 2 diabetes mellitus (T2DM) is a common metabolic disease, affecting nearly 300 million individuals worldwide. T2DM affects over $10 \%$ of adult individuals over 40 years of age in Japan. The continuous increase in the number of patients is a major public health problem worldwide. Loci for rare monogenic forms of diabetes, such as maturity-onset diabetes of the young [1], mitochondrial diabetes [2,3], and Wolfram syndrome [4], have been elucidated in a limited proportion of patients. However, the etiology of sporadic T2DM remains largely unknown. Accumulating epidemiological evidence [5-8] suggests that genetic factors play an important role in the susceptibility to sporadic T2DM, in addition to environmental factors such as obesity, aging, and exercise.

To search for susceptibility gene(s) for sporadic T2DM, genome-wide association studies (GWASs) using single nu- cleotide polymorphism (SNP) markers have been performed. These GWASs and replication studies have found multiple loci, TCF7L2 [9], KCNQ1 [10, 11], and others $[12-18]$, that are associated with susceptibility to T2DM. However, the overall contribution of these SNPs to sporadic T2DM is relatively low; their odds ratio being in the range of 1.1-1.4 [9, 11]. In addition, these associations have not necessarily been replicated in subsequent studies [12-18].

Copy number variations (CNVs) or structural variations, such as deletion or gain of a genomic region, are increasingly recognized as important interindividual genetic variations across the human genome. CNVs account for more nucleotide variation between two individuals than do SNPs [19-21]. Repetitive, multicopy regions, such as segmental duplications and low copy repeats associated with CNV, are regarded as "rearrangement hotspots," and CNV regions are predisposed to the generation of deletion/duplication events [22]. Such repeat-rich regions were recently found to 


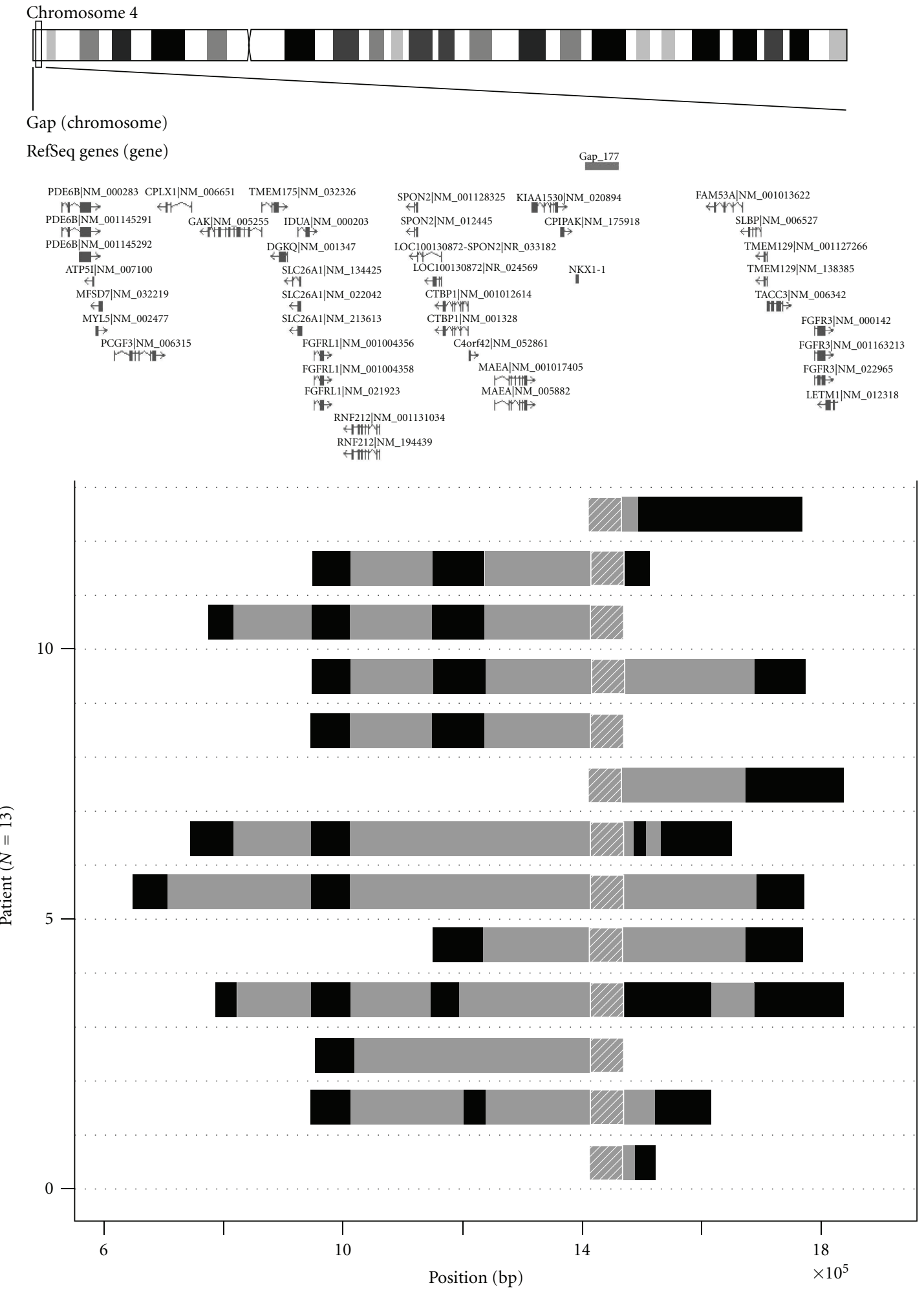

FIGURE 1: Genomic region harboring copy number loss of 1.3-Mb 4p16.3 subtelomeric region in 13 early-onset T2DM patients. Data measured by deCODE/Illumina CNV370K chip were analyzed by the PennCNV program. Genome structure of the 13 patients are aligned as horizontal bars from genome position 550,000 (left) to position 1,850,000 (right). Hatched region at position 1,423,1471,478,646 represents genome gap-177 region. Dark solid horizontal bars represent extent of copy number loss in each T2DM patient. Gray regions between the dark bars represent intervals where copy number loss could not be inferred due to poor probe coverage. Upper map shows ideogram of chromosome 4 and the positions of putative genes in 4p16.3 region described in Database of Genomic Variants (http://projects.tcag.ca/variation/). Position is given relative to NCBI Build 35 for the chromosome 4. 
show 13-fold enrichment of CNVs over the average genomic coverage in a reference assembly [23]. Therefore, CNVs or structural variations are recognized as significant contributors to human genetic disease and disease susceptibility [24].

In the search for susceptibility gene(s) for T2DM genes, we recruited a panel of 100 early-onset Japanese T2DM patients (onset age $<35$ years) and 100 controls, and performed $\mathrm{CNV}$ analysis in the whole genome using the deCODE-Illumina CNV370K BeadChip which focuses on the CNV-rich region of the human genome, followed by validation and characterization using an Agilent region-targeted high-density custom-made oligonucleotide tiling microarray. We found frequent copy number losses within the 1.3$\mathrm{Mb}$ subtelomeric region in a substantial portion of earlyonset Japanese T2DM patients. This region surrounds the genome gap in $4 \mathrm{p} 16.3$, which is rich in multiple low copy repeats.

\section{Materials and Methods}

2.1. Subjects. We considered that the early onset of T2DM reflects the presence of more genetic factors rather than environmental factors. Therefore, we adopted young-onset diabetic patients as case subjects. We studied 100 unrelated Japanese T2DM patients who developed T2DM before 35 years of age. They were recruited at Tohoku University Hospital and affiliated hospitals and medical clinics. Diabetes was diagnosed using the WHO criteria. Type 1 diabetes mellitus was excluded judged from clinical features and existence of anti-GAD (glutamic acid decarboxylase) antibodies or anti-IA-2 (insulinoma-associated antigen-2) antibodies. Patients with diabetes mellitus due to hepatic disease, pancreatic disease, other endocrinological disease, or mitochondrial DNA mutation, or drug-induced diabetes were excluded, judged from laboratory data and clinical history.

We also studied 100 nondiabetic control subjects, using the following criteria: 60 or more years of age, no prior diagnosis of diabetes mellitus, HbA1c less than 6.4\% (where HbAlc (\%) was estimated as an NGSP (National Glycohemoglobin Standardization Program) equivalent value (\%) calculated by the formula HbA1c (\%) = HbA1c (JDS: Japan Diabetes Society value) $(\%)+0.4 \%$, considering the relational expression of HbAlc (JDS) (\%) measured by the previous Japanese standard substance and measurement methods for HbA1c (NGSP) [25] and no family history of T2DM within third-degree relatives, in order to exclude subjects who were more likely to develop diabetes later.

Clinical features available from 100 early-onset T2DM patients and 100 controls are shown in (see Table S1 in Supplementary Material available online at doi: 10.1155/ $2011 / 498460)$. In addition, clinical features of the 13 earlyonset T2DM patients with copy number losses in 4p16.3 are shown separately in Supplementary Table S2 and Supplementary Table S3 in comparison with the rest of 100 earlyonset T2DM patients without copy number loss $(n=87)$.

Genetic analysis of human subjects was approved by the ethics committee of Tohoku University Graduate School of
Medicine. Appropriate informed consent was obtained from all the subjects examined.

2.2. Screening with Whole-Genome CNV BeadChip. We screened the whole genome by CNV analysis using the deCODE-Illumina CNV370K BeadChip (Illumina Infinium system, deCODE genetics, Inc., Iceland), which, in addition to Hap300 SNP marker contents, has CNV probes designed to target the CNV-rich region of the whole genome. The $\mathrm{CNV}$ part of the platform consists of probes covering CNVrich regions of the genome, such as megasatellites (tandem repeats $>500 \mathrm{bp}$ ), duplicons (region flanked by highly homologous segmental duplication $>1 \mathrm{~kb}$ ), unSNPable regions ( $>15 \mathrm{~kb}$ gaps in HapMap SNP map, and $5-15 \mathrm{~kb}$ gaps with $>$ 2SNPs with Hardy-Weinberg failure), and CNVs registered in the Database of Genomic Variants. The CNV part of probe content consists of 15,559 CNV segments covering $190 \mathrm{Mb}$, or $6 \%$ of the human genome. The platform has been tested in 4000 Icelandic and HapMap samples.

Data analysis of the deCODE-Illumina CNV chip was carried out using DosageMiner software developed by deCODE genetics, and loss/gain analysis consisted of the following four steps; (1) intensity normalization and GC content correction, (2) removal of batch effects using principal component analysis, (3) calling of clusters using a Gaussian mixture model, and (4) determination of CNV type using graphical constraints. In brief, CNVs were identified when CNV events stood out in the data, as all sample intensities for $\mathrm{CNV}$ probes should be increased or decreased relative to neighboring probes that are not in a CNV region. To determine deviations in signal intensity we started by normalizing the intensities. The normalized intensities for each color channel were determined by an equation and fit formula developed by deCODE genetics. A stretch with occurrence of more than one marker showing abnormality in the copy number in a consecutive stretch in the genome is considered more likely to be evidence of deletion or gain [26]. We display Supplementary Table to present raw data, that is, $\log _{2}$ ratio measured at each probe for every individual. Raw data at screening step via deCODE/Illumina beads chip for all probes on chromosome $4 \mathrm{p}$ is shown in Supplementary Table S4.

\subsection{High-Density Custom-Made Oligonucleotide Tiling Mi-} croarray Analysis. DNA samples from 13 early-onset T2DM patients and 15 control individuals were subjected to Agilent's high-density custom-made oligonucleotide tiling microarray analysis based on an array comparative genomic hybridization (aCGH) assay. We fabricated a custom-designed microarray targeted to a $1.3-\mathrm{Mb}$ genome region in the subtelomere at 4p16.3 (Chr. 4: 550,000-1,850,000 (NCBI Build 36.1, hg18)) according to previously described methods [27, 28]. In brief, we used the Agilent website (http://earray.chem.agilent.com/earray/) to select and design our custom tiling array; the array consisted of probes 60-mer in size (Agilent Technologies, Santa Clara, CA).

Tiling-aCGH experiments were performed essentially as described previously [29]. In brief, test and reference (NA19000, a Japanese male from HapMap project) genomic 


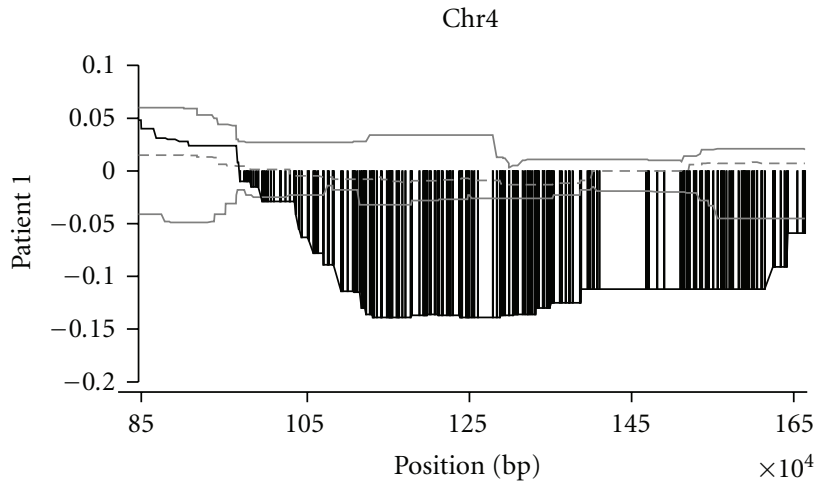

(a)

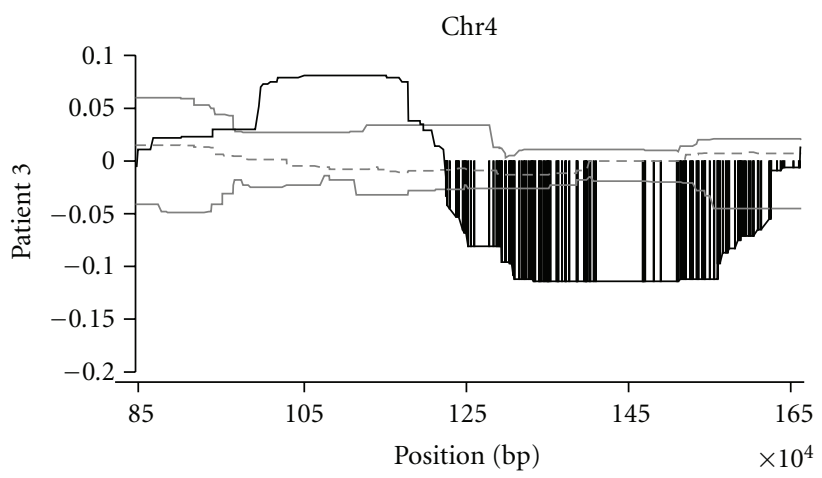

(c)

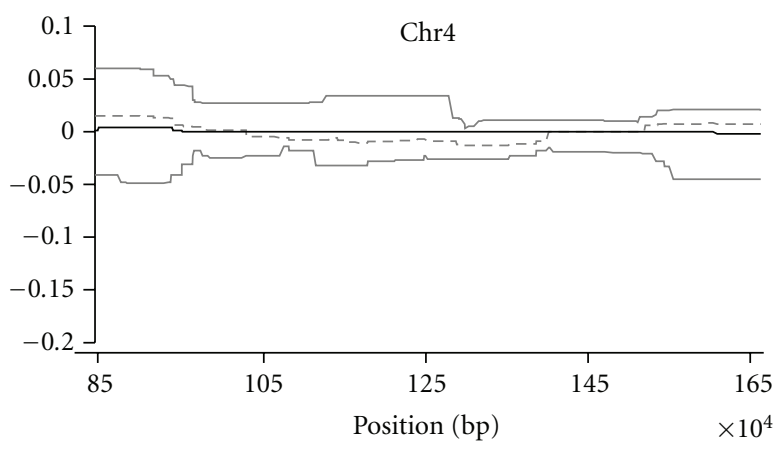

(e)

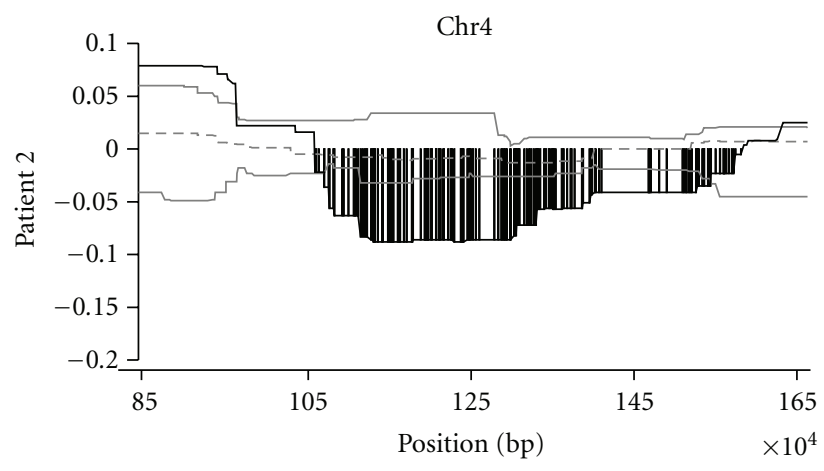

(b)

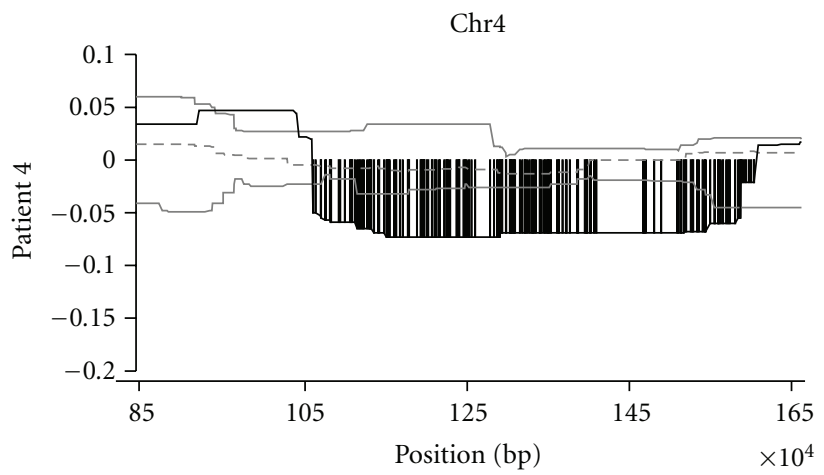

(d)

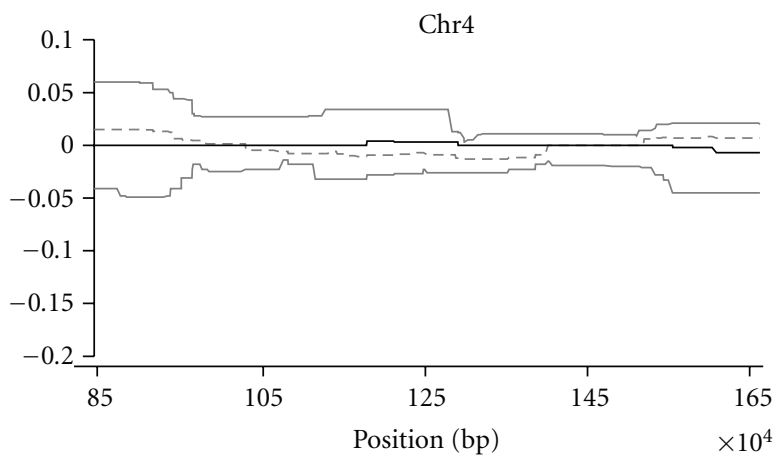

(f)

FIgURE 2: Detailed structure of copy number loss of 1.3-Mb 4p16.3 subtelomeric region resolved by high-density tiling microarray. Log ${ }_{2}$ ratio ( $y$-axis) was plotted using moving average along the genome position ( $x$-axis). Four representative early-onset T2DM patients are shown as Figures 2 (a), 2(b), 2(c), and 2 (d), patient 1, 2, 3, and 4, respectively. For comparison, $\log _{2}$ ratio of the region of two healthy normal individuals is also displayed as Figures 2(e) and 2(f). Dark line represents copy number plot along the genome. Two light lines indicate normal range of average $\log _{2}$ ratios for probes among normal individuals. Dotted line shows median of average $\log _{2}$ ratio among normal individuals. Copy number losses are displayed as gray vertical bar. We defined two copy number classes, that is, "unchanged copy number" and "copy number loss." "Unchanged copy number" was defined when the $\log _{2}$ ratio stays within the mean \pm 1 SD distribution among the normal population. "Copy number loss" was called when the downward-deviation of $\log _{2}$ ratios exceeded a threshold of 1 SD from the median probe ratio.

DNAs (250 ng per sample) were fluorescently labeled with Cy5 (test) and Cy3 (reference) with a ULS Labeling Kit (Agilent Technologies).

For each sample, respective labeling reactions were mixed and then separated prior to hybridizing to each of the arrays. Labeled test and reference DNAs were combined, denatured, preannealed with Cot-1 DNA (Invitrogen) and blocking agent, and then hybridized to the arrays for $24 \mathrm{hr}$ in a rotating oven at $65^{\circ} \mathrm{C}$ and $20 \mathrm{rpm}$ (Agilent Technologies). After hybridization and washes, the arrays were scanned at $3 \mu \mathrm{m}$ resolution with an Agilent G2505C scanner. Images were analyzed with Feature Extraction Software 10.7.3.1 (Agilent Technologies), with the CGH_107_Sep09 protocol for background subtraction and normalization. Detection 


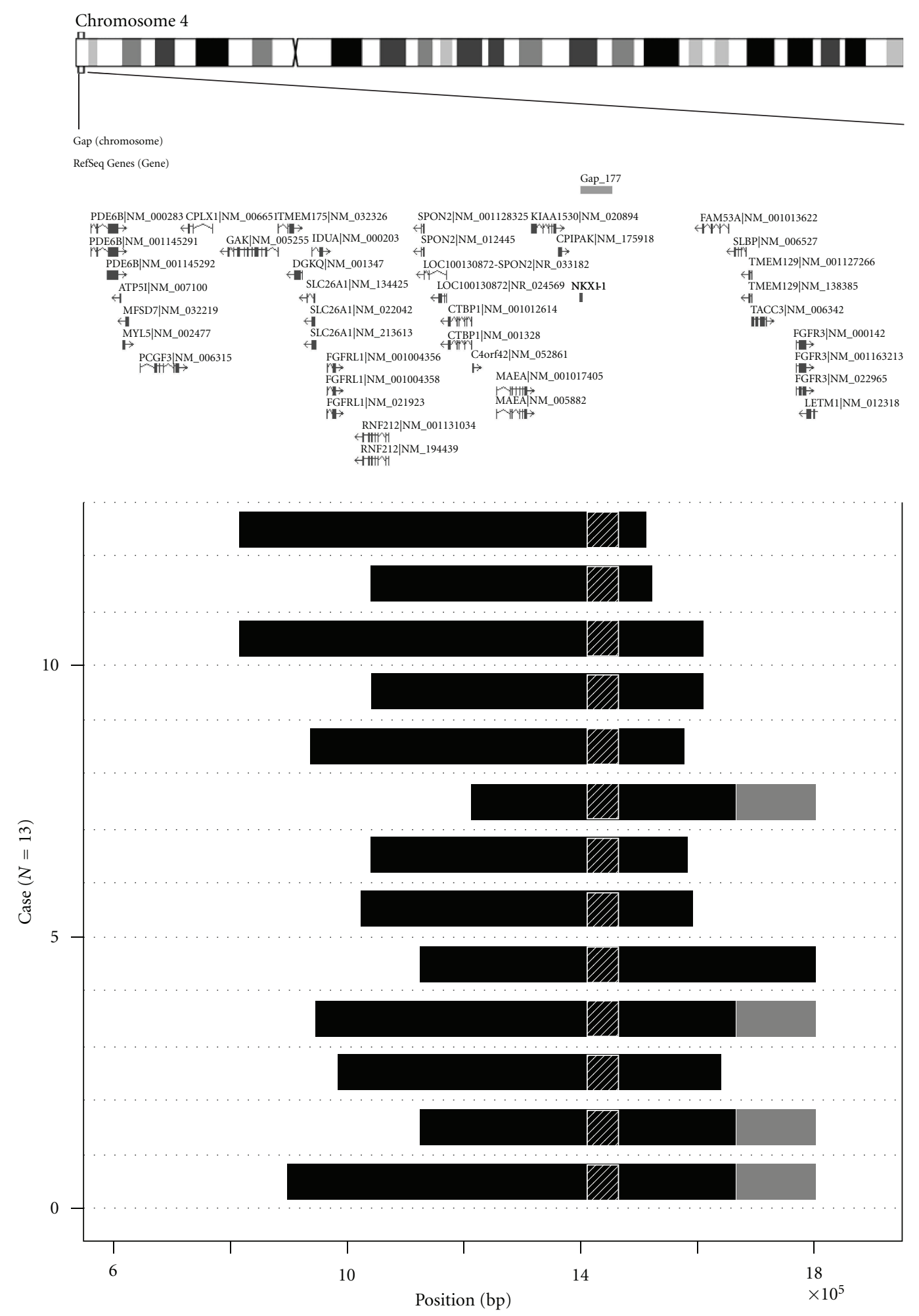

Figure 3: The extent of copy number losses within 1.3-Mb 4p16.3 subtelomeric region in 13 early-onset T2DM patients revealed by high-density oligonucleotide tiling microarray. Dark horizontal bars represent extent of copy number loss region in each patient revealed by Agilent custom tiling array. Genome structure of the 13 patients is aligned as horizontal bars from genome position 550,000 (left) to position $1,850,000$ (right). Hatched region at position 1,423,147-1,478,646 represents genome gap-177 region. Gray regions represent proximal ends of stretch where copy number status was not inferred due to presence of multiple low copy repeats. Upper map shows ideogram of chromosome 4 and the positions of putative genes in 4p16.3 region described in Database of Genomic Variants (http://projects.tcag.ca/variation/). Position is given relative to NCBI Build 35 for the chromosome 4. 
of abnormal copy number, losses, and gains, in a complex multicopy variable region by high-density tiling array was accessed by deviation of probe $\log _{2}$ ratios that exceeded a threshold of 1 SD from the median probe ratio, according to procedures described previously [29-31]. We defined two copy number classes, that is, "unchanged copy number" and "copy number loss." "Unchanged copy number" was defined when the $\log _{2}$ ratio stays within the mean \pm 1 SD distribution among the normal population. "Copy number loss" was called when the downward-deviation of $\log _{2}$ ratios exceeded a threshold of 1 SD from the median probe ratio. Raw data, that is, $\log _{2}$ ratio measured at each probe for every individual obtained by high-density custom-made tilling array analysis is shown in Supplementary Table S5.

\section{Results}

In searching for CNVs associated with early-onset T2DM, we screened the whole genome by $\mathrm{CNV}$ analysis using the deCODE-Illumina CNV370K BeadChip in 100 earlyonset Japanese T2DM patients and 100 controls. We found four CNVs that fulfilled our screening criteria: (1) reliable $\mathrm{CNV}$ (size over $50-\mathrm{Kb}$, consisting of over 50 consecutive probes), (2) the association being statistically significant by Fisher's exact test which was accompanied by Bonferroni's correction. Four candidate $\mathrm{CNV}$ that fulfilled the criteria; that is, CNV on 4p16.3 $\left(P=6.75 \times 10^{-3}\right.$ by Fisher's exact test, $P<.05$ after Bonferroni's correction), CNV on $16 \mathrm{p} 13.3$ $\left(P=3.44 \times 10^{-4}\right.$ by Fisher's exact test, $P<.05$ after Bonferroni's correction), $\mathrm{CNV}$ on $16 \mathrm{q} 24.3\left(P=9.65 \times 10^{-3}\right.$ by Fisher's exact test, $P<.05$ after Bonferroni's correction), and CNV on $19 \mathrm{p} 13.3\left(P=1.61 \times 10^{-4}\right.$ by Fisher's exact test, $P<.05$ after Bonferroni's correction). Of these CNVs listed, the CNV on 4 p16.3 was intriguingly found to be located right on the genome gap-177 region on $4 \mathrm{p}$ subtelomere. At this candidate CNV on 4p16.3, 13 out of 100 T2DM patients displayed $\mathrm{CN}$-loss around this CNV marker, compared with 1 out of 100 control samples. Because of its unique overlap with genome gap structure, we focused on this CNV region for further analysis.

Figure 1 shows the pattern of alterations in copy number loss observed among the 100 early-onset Japanese 2DM patients. Thirteen patients displayed copy number losses around the gap in the $4 \mathrm{p} 16.3$ subtelomere whereas only one of 100 control samples showed copy number losses in this region. The association was statistically significant by Fisher's exact test $\left(P=6.75 \times 10^{-3}\right.$, OR $=14.7,95 \%$ confidence interval 3.02-72.3). We observed two copy number classes, that is, "unchanged copy number" and "copy number loss" at $1.3-\mathrm{Mb}$ region of chromosome 4 p16.3 in our diabetic or control populations. The latter was found frequently observed among early-onset T2DM patients at the 4p16.3 subtelomere. The position, length, or pattern of deletion between one copy number loss in control and 13 copy number losses in T2DM patients were not apparently distinct, although the case number is too small to draw meaningful conclusion.

To verify the CNV BeadChip results, we analyzed copy number changes along the $1.3-\mathrm{Mb}$ region in the subtelomere surrounding the genome gap of 4 p16.3 using a high-density custom-made oligonucleotide tiling microarray. We used peripheral blood DNA of the 13 early-onset T2DM patients, identified, and 15 control healthy individuals. Again, we found frequent copy number losses in regions around the genome gap in all the 13 early-onset T2DM patients, whereas none of 15 healthy individuals showed copy number losses.

Figure 2 shows detailed structure of copy number losses in four representative early-onset Japanese T2DM patients with copy number losses, in the $1.3-\mathrm{Mb}$ region in the subtelomere (patients 1, 2, 3, 4; in Figures 2(a), 2(b), 2(c), and $2(\mathrm{~d})$, resp.). Individual copy number plots using moving average $(y$-axis) versus distance along the chromosome $(x$ axis) are shown. As a comparison, copy number plots of healthy individuals who did not exhibit copy number alterations in the region are also shown (Figures 2(e) and 2(f)).

Figure 3 shows genomic copy number losses in all the 13 early-onset T2DM patients. High-density tiling custommade microarray showed segmental losses in the subtelomeric region of 4 p16.3 in all the 13 patients. Genomic copy number losses in these patients were clustered around a gap region in the 4 p 16.3 subtelomeric region.

\section{Discussion}

Our initial genome-wide screening with deCODE-Illumina CNV370K BeadChip for association with early-onset T2DM revealed losses in the subtelomeric region of $4 \mathrm{p} 16.3$. Subsequent high-density custom-made oligonucleotide tiling microarray verified copy number losses in this region.

It is worthy to note that most patients with copy number losses were treated with insulin injection. Urine C-peptide reactivity was not significantly different between the two groups, and only few patients underwent glucagon challenge test, thus, the data are too limited to infer the function of insulin secretion. We did not observe significant differences between two groups as to age of onset, body mass index, postprandial plasma glucose levels, or HbA1c levels. Fasting immunoreactive insulin levels were examined in 5 patients with copy number losses and 14 patients without copy number loss. HOMA-R in the former patients was $6.1 \pm$ 6.8 (mean $\pm \mathrm{SD}$ ) and that in the latter patients was $5.5 \pm 6.0$ (mean $\pm \mathrm{SD}$ ); these values were not significantly different. Incidence of dyslipidemia, hypertension, diabetic retinopathy, nephropathy, or neuropathy was not different between the two groups (Supplementary Tables S2, S3). Further investigation of a large panel of patients would be necessary to clarify any clinical differences that might be present between the two groups.

The current map of CNV in the human genome reported in the existing databases is far from complete [32]. Increasing numbers of CNVs have recently been identified around repetitive sequences such as segmental duplications or low copy repeats. In fact, these repeat-rich regions were found to be 13 -fold enriched in $\mathrm{CNV}$ over the average genomic coverage in the reference assembly [23]. Probes for conventional genome-wide SNP genotyping platforms are likely to be underrepresented; that is, only $25 \%$ and $40 \%$ of CNV are 
TABLe 1: Putative genes located within 1.3-Mb region in 4p16.3 subtelomere.

\begin{tabular}{|c|c|c|c|c|c|}
\hline Start & End & $\begin{array}{l}\text { Cytogenetic } \\
\text { location }\end{array}$ & Symbol & Description & Model evidence \\
\hline 609,373 & 654,571 & $4 \mathrm{p} 16.3$ & PDE6B & $\begin{array}{l}\text { Phosphodiesterase 6B, cGMP-specific, rod, beta } \\
\text { (congenital stationary night blindness } 3, \\
\text { autosomal dominant) }\end{array}$ & Best RefSeq \\
\hline 656,225 & 658,122 & $4 \mathrm{p} 16.3$ & ATP5I & $\begin{array}{l}\text { ATP synthase, } \mathrm{H}+\text { transporting, mitochondrial F0 } \\
\text { complex, subunit E }\end{array}$ & Best RefSeq \\
\hline 661,711 & 665,817 & $4 \mathrm{p} 16.3$ & MYL5 & Myosin, light chain 5 , regulatory & Best RefSeq \\
\hline 665,618 & 672,973 & $4 \mathrm{p} 16.3$ & MFSD7 & Major facilitator superfamily domain containing 7 & mRNA \\
\hline 689,573 & 754,428 & $4 \mathrm{p} 16.3$ & PCGF3 & Polycomb group ring finger 3 & Best RefSeq \\
\hline 719,829 & 721,544 & $4 \mathrm{p} 16.3$ & LOC100128084 & Hypothetical protein LOC100128084 & mRNA \\
\hline 764,588 & 765,632 & $4 \mathrm{p} 16.3$ & LOC100129917 & Hypothetical protein LOC100129917 & mRNA \\
\hline 768,745 & 809,945 & $4 \mathrm{p} 16.3$ & CPLX1 & Complexin 1 & mRNA \\
\hline 833,065 & 916,174 & $4 \mathrm{p} 16.3$ & GAK & Cyclin $\mathrm{G}$ associated kinase & mRNA \\
\hline 916,262 & 942,444 & $4 \mathrm{p} 16.3$ & TMEM175 & Transmembrane protein 175 & Best RefSeq \\
\hline 942,675 & 957,344 & $4 \mathrm{p} 16.3$ & DGKQ & Diacylglycerol kinase, theta $110 \mathrm{kDa}$ & Best RefSeq \\
\hline 962,861 & 977,224 & $4 \mathrm{p} 16.3$ & SLC26A1 & $\begin{array}{l}\text { Solute carrier family } 26 \text { (sulfate transporter), } \\
\text { member } 1\end{array}$ & Best RefSeq \\
\hline 970,785 & 988,317 & $4 \mathrm{p} 16.3$ & IDUA & Iduronidase, alpha-L- & mRNA \\
\hline 995,610 & $1,010,686$ & $4 \mathrm{p} 16.3$ & FGFRL1 & Fibroblast growth factor receptor-like 1 & Best RefSeq \\
\hline $1,044,654$ & $1,045,386$ & $4 \mathrm{p} 16.3$ & LOC100132787 & Hypothetical protein LOC100132787 & mRNA \\
\hline $1,055,269$ & $1,097,350$ & $4 \mathrm{p} 16.3$ & RNF212 & Ring finger protein 212 & Best RefSeq \\
\hline $1,116,050$ & $1,129,814$ & $4 \mathrm{p} 16.3$ & LOC100133135 & Hypothetical protein LOC100133135 & Protein \\
\hline $1,135,541$ & $1,135,957$ & $4 \mathrm{p} 16.3$ & FLJ35816 & FLJ35816 protein & Protein \\
\hline $1,149,293$ & $1,149,712$ & $4 \mathrm{p} 16.3$ & LOC100131106 & Hypothetical protein LOC100131106 & Protein \\
\hline $1,150,723$ & $1,156,597$ & $4 \mathrm{p} 16.3$ & SPON2 & Spondin 2, extracellular matrix protein & Best RefSeq \\
\hline $1,184,431$ & $1,185,198$ & $4 \mathrm{p} 16.3$ & LOC100130872 & Hypothetical protein LOC100130872 & mRNA \\
\hline $1,195,228$ & $1,232,908$ & $4 \mathrm{p} 16.3$ & СТBP1 & C-terminal binding protein 1 & Best RefSeq \\
\hline $1,234,177$ & $1,236,616$ & $4 \mathrm{p} 16.3$ & C4orf42 & Chromosome 4 open reading frame 42 & Best RefSeq \\
\hline $1,273,672$ & $1,323,925$ & $4 \mathrm{p} 16.3$ & MAEA & Macrophage erythroblast attacher & Best RefSeq \\
\hline $1,331,104$ & $1,371,732$ & $4 \mathrm{p} 16.3$ & KIAA1530 & KIAA1530 & mRNA \\
\hline $1,375,340$ & $1,379,782$ & $4 \mathrm{p} 16.3$ & CRIPAK & Cysteine-rich PAK1 inhibitor & mRNA \\
\hline $1,380,072$ & $1,392,453$ & $4 \mathrm{p} 16.3$ & NKX1-1 & NK1 homeobox 1 & Protein \\
\hline $1,484,196$ & $1,519,086$ & $4 \mathrm{p} 16.3$ & LOC100133199 & Similar to RE32881p & mRNA \\
\hline $1,611,605$ & $1,655,516$ & $4 \mathrm{p} 16.3$ & FAM53A & Family with sequence similarity 53 , member A & Best RefSeq \\
\hline $1,664,325$ & $1,683,828$ & $4 \mathrm{p} 16.3$ & SLBP & Stem-loop (histone) binding protein & Best RefSeq \\
\hline $1,687,477$ & $1,692,882$ & $4 \mathrm{p} 16.3$ & TMEM129 & Transmembrane protein 129 & Best RefSeq \\
\hline $1,693,062$ & $1,716,696$ & $4 \mathrm{p} 16.3$ & TACC3 & $\begin{array}{l}\text { Transforming, acidic coiled-coil containing } \\
\text { protein } 3\end{array}$ & Best RefSeq \\
\hline $1,765,421$ & $1,780,396$ & $4 \mathrm{p} 16.3$ & FGFR3 & $\begin{array}{l}\text { Fibroblast growth factor receptor } 3 \\
\text { (achondroplasia, thanatophoric dwarfism) }\end{array}$ & Best RefSeq \\
\hline $1,784,558$ & $1,827,772$ & $4 \mathrm{p} 16.3$ & LETM1 & $\begin{array}{l}\text { Leucine zipper-EF-hand containing } \\
\text { transmembrane protein } 1\end{array}$ & Best RefSeq \\
\hline
\end{tabular}

covered by the HumanHap300 and HumanHap550 platform, respectively [23]. These recent findings may partly explain why earlier genome-wide association studies for CNVs in the T2DM population failed to detect CNV loci being strongly associated with T2DM $[20,21,33,34]$.

We found copy number losses among early-onset Japanese T2DM patients in a region surrounding a genome gap (gap-177) in the subtelomere of chromosome $4 \mathrm{p} 16.3$. The physical map of the human genome still contains a significant number of genome gaps; over 300 gaps still remain in the human draft genome sequence [35] that are considered inaccessible by most existing genotyping and sequencing technologies [36]. These gap regions are estimated to harbor $\sim 1000$ genes, which comprise approximately $5 \%$ of 
the human genome $(\sim 200 \mathrm{Mb})$. In particular, they are abundant in subtelomeres and pericentromeric regions of chromosomes $[37,38]$. Some of these gaps are thought to be susceptible sites for mediating meiotic recombination and are also susceptibility sites for break points for deletions $[39,40]$.

Many CNVs were recently identified in repeat-rich regions [41], which are predisposed to the generation of deletion/duplication events [22]. It is intriguing to note that the locus-specific mutation rates for $\mathrm{CNV}$ or structural rearrangements were estimated to be between $10^{-6}$ and $10^{-4}$ : two to four orders of magnitude greater than nucleotidespecific rates for base substitutions or point mutations [19].

The deleted CNV region found in the present study and its flanking region contained 34 genes (Table 1). Gene(s) in this region may predispose to T2DM.

Genes involved in the glucose-induced insulin secretion cascade of pancreatic beta-cells are located in the region, such as ATP5I (ATP synthase, $\mathrm{H}^{+}$transporting, mitochondrial $\mathrm{F}_{0}$ complex, subunit E) [42, 43], CPLX1 (complexin 1) [44, 45], GAK (cyclin G associated kinase) in association with CDK5 (cyclin-dependent protein kinases 5) [46-50], and CRIPAK (cysteine-rich PAK1 inhibitor), which is a negative regulator of PAK1 (p21 protein (Cdc42/Rac-) activated kinase 1) [51, 52].

FGFR3 (fibroblast growth factor receptor 3) and FGFRL1 (fibroblast growth factor receptor-like 1) are suggested to be involved in pancreatic development and differentiation [5356]. TACC3 (transforming, acidic coiled-coil containing protein 3) may be involved in embryonic development including the pancreas $[57,58]$.

CTBP1 (C-terminal binding protein 1) and MAEA (macrophage erythroblast attacher) have effects on adipose tissue functions $[59,60]$, which may lead to insulin resistance. NKX1-1(NK1 homeobox 1) may cause insulin resistance with a function in the maintenance of energy homeostasis [61].

Recently, through whole-genome screening of a copy number variation using a CNV BeadChip and real-time quantitative polymerase chain reaction (qPCR), Kato et al. identified a segmental copy number gain within the $40-\mathrm{kb}$ region on 10p15.3 subtelomere in patients of sporadic amyotrophic lateral sclerosis (SALS) [62]. They demonstrated the copy number gain in 46 out of 83 SALS patients, as compared with 10 out of 99 controls. The copy number gain region they identified is rather small $(40-\mathrm{kb})$ and harbored two genes encoding isopentenyl diphosphate isomerase 1 (IDI1) and IDI2. Thus, they suggested the copy number gain in the region of these genes may play a significant role in the pathogenesis of SALS. The present study share a similar genome abnormality in that we found frequent copy number alterations in subtelomere region among sporadic adult-onset disease of unknown cause. The copy number alterations we identified here in early-onset T2DM were copy number losses on different chromosome subtelomere (4p16.3) and the size is rather large (up to $1.3-\mathrm{Mb}$ ). In our case, we suspect that multiple genes in the region may be involved in diabetes pathogenesis through impairments caused by copy number losses.

\section{Conclusion}

These results suggested that copy number losses of the candidate genes in the deleted region surrounding the genome gap in 4p16.3 may play significant roles in the etiology of T2DM. Further functional study, as well as investigation of $4 \mathrm{p} 16.3$ loss in a large panel of early-onset T2DM patients in different ethnic populations and geographical regions, is warranted.

\section{Acknowledgment}

This paper was supported by a Grant-in-Aid for Scientific Research to Y. Oka (H19-genome-005) from the Ministry of Health, Labor, and Welfare of Japan, and was also supported by a grant from the Global-COE Programs for "Network Medicine" to Y. Oka and H. Katagiri from the Ministry of Education, Culture, Sports, Science, and Technology of Japan.

\section{References}

[1] T. M. Frayling, J. C. Evans, M. P. Bulman et al., "beta-cell genes and diabetes: molecular and clinical characterization of mutations in transcription factors," Diabetes, vol. 50, supplement 1 , pp. S94-S100, 2001.

[2] J. M. van den Ouweland, H. H. Lemkes, W. Ruitenbeek et al., "Mutation in mitochondrial tRNA(Leu)(UUR) gene in a large pedigree with maternally transmitted type II diabetes mellitus and deafness," Nature Genetics, vol. 1, no. 5, pp. 368-371, 1992.

[3] Y. Oka, H. Katagiri, Y. Yazaki, T. Murase, and T. Kobayashi, "Mitochondrial gene mutation in islet-cell-antibody-positive patients who were initially non-insulin-dependent diabetics," The Lancet, vol. 342, no. 8870, pp. 527-528, 1993.

[4] H. Inoue, Y. Tanizawa, J. Wasson et al., "A gene encoding a transmembrane protein is mutated in patients with diabetes mellitus and optic atrophy (Wolfram syndrome)," Nature Genetics, vol. 20, no. 2, pp. 143-148, 1998.

[5] J. Kaprio, J. Tuomilehto, M. Koskenvuo et al., "Concordance for Type 1 (insulin-dependent) and Type 2 (non-insulindependent) diabetes mellitus in a population-based cohort of twins in Finland," Diabetologia, vol. 35, no. 11, pp. 1060-1067, 1992.

[6] P. Poulsen, K. O. Kyvik, A. Vaag, and H. Beck-Nielsen, "Heritability of type II (non-insulin-dependent) diabetes mellitus and abnormal glucose tolerance-a population-based twin study," Diabetologia, vol. 42, no. 2, pp. 139-145, 1999.

[7] J. B. Meigs, L. A. Cupples, and P. W. Wilson, "Parental transmission of type 2 diabetes: the Framingham Offspring Study," Diabetes, vol. 49, no. 12, pp. 2201-2207, 2000.

[8] C. F. Weijnen, S. S. Rich, J. B. Meigs, A. S. Krolewski, and J. H. Warram, "Risk of diabetes in siblings of index cases with Type 2 diabetes: implications for genetic studies," Diabetic Medicine, vol. 19, no. 1, pp. 41-50, 2002.

[9] S. F. Grant, G. Thorleifsson, I. Reynisdottir et al., "Variant of transcription factor 7-like 2 (TCF7L2) gene confers risk of type 2 diabetes," Nature Genetics, vol. 38, no. 3, pp. 320-323, 2006.

[10] H. Unoki, A. Takahashi, T. Kawaguchi et al., "SNPs in KCNQ1 are associated with susceptibility to type 2 diabetes in East Asian and European populations," Nature Genetics, vol. 40, no. 9, pp. 1098-1102, 2008. 
[11] K. Yasuda, K. Miyake, Y. Horikawa et al., "Variants in KCNQ1 are associated with susceptibility to type 2 diabetes mellitus," Nature Genetics, vol. 40, no. 9, pp. 1092-1097, 2008.

[12] R. Saxena, B. F. Voight, V. Lyssenko et al., "Genome-wide association analysis identifies loci for type 2 diabetes and triglyceride levels," Science, vol. 316, no. 5829, pp. 1331-1336, 2007.

[13] L. J. Scott, K. L. Mohlke, L. L. Bonnycastle et al., "A genomewide association study of type 2 diabetes in Finns detects multiple susceptibility variants," Science, vol. 316, no. 5829, pp. 1341-1345, 2007.

[14] R. Sladek, G. Rocheleau, J. Rung et al., "A genome-wide association study identifies novel risk loci for type 2 diabetes," Nature, vol. 445, no. 7130, pp. 881-885, 2007.

[15] V. Steinthorsdottir, G. Thorleifsson, I. Reynisdottir et al., "A variant in CDKAL1 influences insulin response and risk of type 2 diabetes," Nature Genetics, vol. 39, no. 6, pp. 770-775, 2007.

[16] E. Zeggini, M. N. Weedon, C. M. Lindgren et al., "Replication of genome-wide association signals in UK samples reveals risk loci for type 2 diabetes," Science, vol. 316, no. 5829, pp. 13361341, 2007.

[17] I. Prokopenko, M. I. McCarthy, and C. M. Lindgren, "Type 2 diabetes: new genes, new understanding," Trends in Genetics, vol. 24, no. 12, pp. 613-621, 2008.

[18] E. Zeggini, L. J. Scott, R. Saxena et al., "Meta-analysis of genome-wide association data and large-scale replication identifies additional susceptibility loci for type 2 diabetes," Nature Genetics, vol. 40, no. 5, pp. 638-645, 2008.

[19] J. R. Lupski, "Genomic rearrangements and sporadic disease," Nature Genetics, vol. 39, no. 7s, pp. S43-S47, 2007.

[20] D. F. Conrad, D. Pinto, R. Redon et al., "Origins and functional impact of copy number variation in the human genome," Nature, vol. 464, no. 7289, pp. 704-712, 2010.

[21] H. Park, J. I. Kim, Y. S. Ju et al., "Discovery of common Asian copy number variants using integrated high-resolution array CGH and massively parallel DNA sequencing," Nature Genetics, vol. 42, no. 5, pp. 400-405, 2010.

[22] A. J. Sharp, D. P. Locke, S. D. McGrath et al., "Segmental duplications and copy-number variation in the human genome," American Journal of Human Genetics, vol. 77, no. 1, pp. 78-88, 2005.

[23] G. M. Cooper, T. Zerr, J. M. Kidd, E. E. Eichler, and D. A. Nickerson, "Systematic assessment of copy number variant detection via genome-wide SNP genotyping," Nature Genetics, vol. 40, no. 10, pp. 1199-1203, 2008.

[24] R. Redon, S. Ishikawa, K. R. Fitch et al., "Global variation in copy number in the human genome," Nature, vol. 444, no. 7118, pp. 444-454, 2006.

[25] Y. Seino, K. Nanjo, N. Tajima et al., "Report of the committee on the classification and diagnostic criteria of diabetes mellitus," Journal of the Japan Diabetes Society, vol. 53, no. 6, pp. 450-467, 2010.

[26] H. Stefansson, D. Rujescu, S. Cichon et al., "Large recurrent microdeletions associated with schizophrenia," Nature, vol. 455, no. 7210, pp. 232-236, 2008.

[27] M. T. Barrett, A. Scheffer, A. Ben-Dor et al., "Comparative genomic hybridization using oligonucleotide microarrays and total genomic DNA," Proceedings of the National Academy of Sciences of the United States of America, vol. 101, no. 51, pp. 17765-17770, 2004.
[28] G. H. Perry, A. Ben-Dor, A. Tsalenko et al., "The fine-scale and complex architecture of human copy-number variation," American Journal of Human Genetics, vol. 82, no. 3, pp. 685695, 2008.

[29] A. J. de Smith, A. Tsalenko, N. Sampas et al., "Array CGH analysis of copy number variation identifies 1284 new genes variant in healthy white males: implications for association studies of complex diseases," Human Molecular Genetics, vol. 16, no. 23, pp. 2783-2794, 2007.

[30] A. J. Sharp, S. Hansen, R. R. Selzer et al., "Discovery of previously unidentified genomic disorders from the duplication architecture of the human genome," Nature Genetics, vol. 38, no. 9, pp. 1038-1042, 2006.

[31] J. R. Lupski, "Genomic disorders ten years on," Genome Medicine, vol. 1, no. 4, p. 42, 2009.

[32] I. Ionita-Laza, A. J. Rogers, C. Lange, B. A. Raby, and C. Lee, "Genetic association analysis of copy-number variation (CNV) in human disease pathogenesis," Genomics, vol. 93, no. 1, pp. 22-26, 2009.

[33] C. Shtir, R. Pique-Regi, K. Siegmund, J. Morrison, F. Schumacher, and P. Marjoram, "Copy number variation in the Framingham Heart Study," BioMed Central Proceedings, vol. 3, supplement 7, p. S133, 2009.

[34] The Wellcome Trust Case Control Consortium, "Genomewide association study of CNVs in 16,000 cases of eight common diseases and 3,000 shared controls," Nature, vol. 464, no. 7289, pp. 713-720, 2010.

[35] International Human Genome Sequencing Consortium, "Finishing the euchromatic sequence of the human genome," Nature, vol. 431, no. 7011, pp. 931-945, 2004.

[36] E. E. Eichler, J. Flint, G. Gibson et al., "Missing heritability and strategies for finding the underlying causes of complex disease," Nature Reviews Genetics, vol. 11, no. 6, pp. 446-450, 2010.

[37] C. M. Lese, J. A. Fantes, H. C. Riethman, and D. H. Ledbetter, "Characterization of physical gap sizes at human telomeres," Genome Research, vol. 9, no. 9, pp. 888-894, 1999.

[38] E. E. Eichler, R. A. Clark, and X. She, "An assessment of the sequence gaps: unfinished business in a finished human genome," Nature Reviews Genetics, vol. 5, no. 5, pp. 345-354, 2004.

[39] K. Devriendt and J. R. Vermeesch, "Chromosomal phenotypes and submicroscopic abnormalities," Human genomics, vol. 1, no. 2, pp. 126-133, 2004.

[40] G. Van Buggenhout, C. Melotte, B. Dutta et al., "Mild WolfHirschhorn syndrome: micro-array CGH analysis of atypical 4 p16.3 deletions enables refinement of the genotypephenotype map," Journal of Medical Genetics, vol. 41, no. 9, pp. 691-698, 2004.

[41] A. Itsara, G. M. Cooper, C. Baker et al., "Population analysis of large copy number variants and hotspots of human genetic disease," American Journal of Human Genetics, vol. 84, no. 2, pp. 148-161, 2009.

[42] D. A. Swartz, E. I. Park, W. J. Visek, and J. Kaput, "The e subunit gene of murine FF-ATP synthase. Genomic sequence, chromosomal mapping, and diet regulation," Journal of Biological Chemistry, vol. 271, no. 34, pp. 20942-20948, 1996.

[43] T. Hayakawa, M. Noda, K. Yasuda et al., "Ethidium bromideinduced inhibition of mitochondrial gene transcription suppresses glucose-stimulated insulin release in the mouse pancreatic beta-cell cell line betaHC9," Journal of Biological Chemistry, vol. 273, no. 32, pp. 20300-20307, 1998. 
[44] A. Abderrahmani, G. Niederhauser, V. Plaisance et al., "Complexin I regulates glucose-induced secretion in pancreatic beta-cells," Journal of Cell Science, vol. 117, no. 11, pp. 22392247, 2004.

[45] M. Dubois, P. Vacher, B. Roger et al., "Glucotoxicity inhibits late steps of insulin exocytosis," Endocrinology, vol. 148, no. 4, pp. 1605-1614, 2007.

[46] Y. Kanaoka, S. H. Kimura, I. Okazaki, M. Ikeda, and H. Nojima, "GAK: a cyclin G associated kinase contains a tensin/ auxilin-like domain," Federation of European Biochemical Societies Letters, vol. 402, no. 1, pp. 73-80, 1997.

[47] S. H. Kimura, H. Tsuruga, N. Yabuta, Y. Endo, and H. Nojima, "Structure, expression, and chromosomal localization of human GAK," Genomics, vol. 44, no. 2, pp. 179-187, 1997.

[48] F. Y. Wei, K. Nagashima, T. Ohshima et al., "Cdk5-dependent regulation of glucose-stimulated insulin secretion," Nature Medicine, vol. 11, no. 10, pp. 1104-1108, 2005.

[49] M. Ubeda, J. M. Rukstalis, and J. F. Habener, "Inhibition of cyclin-dependent kinase 5 activity protects pancreatic beta cells from glucotoxicity," Journal of Biological Chemistry, vol. 281, no. 39, pp. 28858-28864, 2006.

[50] K. Kitani, S. Oguma, T. Nishiki et al., "A Cdk5 inhibitor enhances the induction of insulin secretion by exendin-4 both in vitro and in vivo," Journal of Physiological Sciences, vol. 57, no. 4, pp. 235-239, 2007.

[51] A. H. Talukder, Q. Meng, and R. Kumar, "CRIPak, a novel endogenous Pak1 inhibitor,” Oncogene, vol. 25, no. 9, pp. 1311-1319, 2006.

[52] Z. Wang, E. Oh, and D. C. Thurmond, "Glucose-stimulated Cdc42 signaling is essential for the second phase of insulin secretion," Journal of Biological Chemistry, vol. 282, no. 13, pp. 9536-9546, 2007.

[53] M. Wiedemann and B. Trueb, "Characterization of a novel protein (FGFRL1) from human cartilage related to FGF receptors," Genomics, vol. 69, no. 2, pp. 275-279, 2000.

[54] A. A. Hardikar, B. Marcus-Samuels, E. Geras-Raaka, B. M. Raaka, and M. C. Gershengorn, "Human pancreatic precursor cells secrete FGF2 to stimulate clustering into hormoneexpressing islet-like cell aggregates," Proceedings of the National Academy of Sciences of the United States of America, vol. 100, no. 12, pp. 7117-7122, 2003.

[55] S. Arnaud-Dabernat, M. Kritzik, A. G. Kayali et al., "FGFR3 is a negative regulator of the expansion of pancreatic epithelial cells," Diabetes, vol. 56, no. 1, pp. 96-106, 2007.

[56] S. D. Gerber, F. Steinberg, M. Beyeler, P. M. Villiger, and B. Trueb, "The murine Fgfrll receptor is essential for the development of the metanephric kidney," Developmental Biology, vol. 335, no. 1, pp. 106-119, 2009.

[57] C. M. Sadek, S. Jalaguier, E. P. Feeney et al., "Isolation and characterization of AINT: a novel ARNT interacting protein expressed during murine embryonic development," Mechanisms of Development, vol. 97, no. 1-2, pp. 13-26, 2000.

[58] C. M. Sadek, M. Pelto-Huikko, M. Tujague, K. R. Steffensen, M. Wennerholm, and J. A. Gustafsson, "TACC3 expression is tightly regulated during early differentiation," Gene Expression Patterns, vol. 3, no. 2, pp. 203-211, 2003.

[59] J. Qiu, Y. H. Ni, R. H. Chen et al., "Gene expression profiles of adipose tissue of obese rats after central administration of neuropeptide Y-Y5 receptor antisense oligodeoxynucleotides by cDNA microarrays," Peptides, vol. 29, no. 11, pp. 20522060, 2008.

[60] C. Vernochet, S. B. Peres, K. E. Davis et al., "C/EBPalpha and the corepressors $\mathrm{CtBP} 1$ and $\mathrm{CtBP} 2$ regulate repression of select visceral white adipose genes during induction of the brown phenotype in white adipocytes by peroxisome proliferatoractivated receptor gamma agonists," Molecular and Cellular Biology, vol. 29, no. 17, pp. 4714-4728, 2009.

[61] R. Simon, T. Lufkin, and A. D. Bergemann, "Homeobox gene Sax2 deficiency causes an imbalance in energy homeostasis," Developmental Dynamics, vol. 236, no. 10, pp. 2792-2799, 2007.

[62] T. Kato, M. Emi, H. Sato et al., "Segmental copy-number gain within the region of isopentenyl diphosphate isomerase genes in sporadic amyotrophic lateral sclerosis," Biochemical and Biophysical Research Communications, vol. 402, no. 2, pp. 438-442, 2010. 


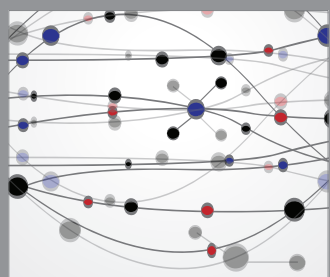

The Scientific World Journal
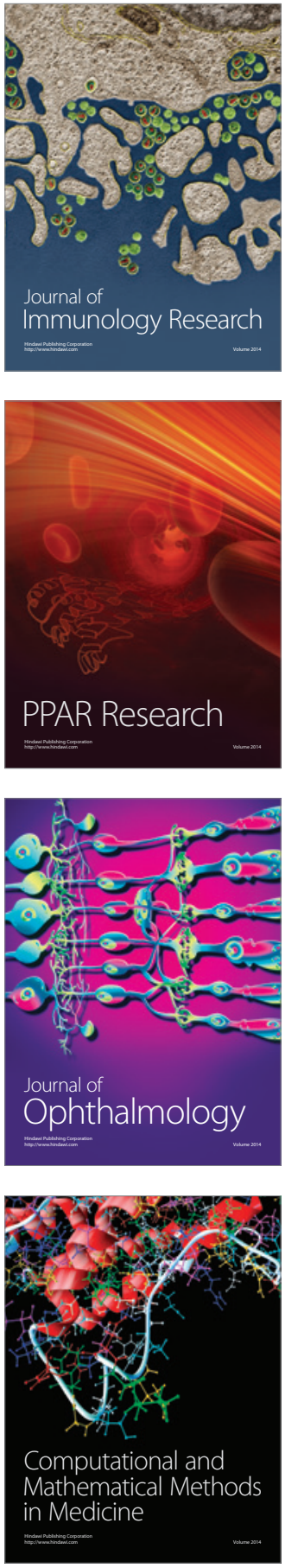

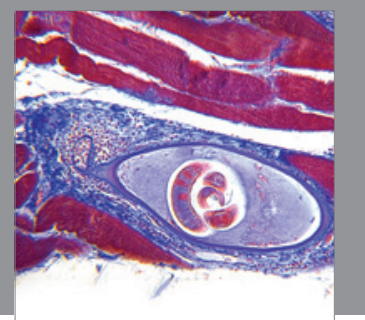

Gastroenterology

Research and Practice
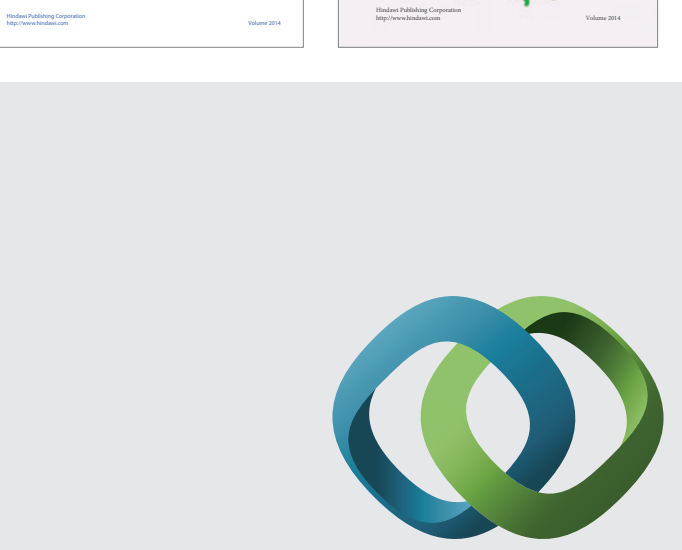

\section{Hindawi}

Submit your manuscripts at

http://www.hindawi.com
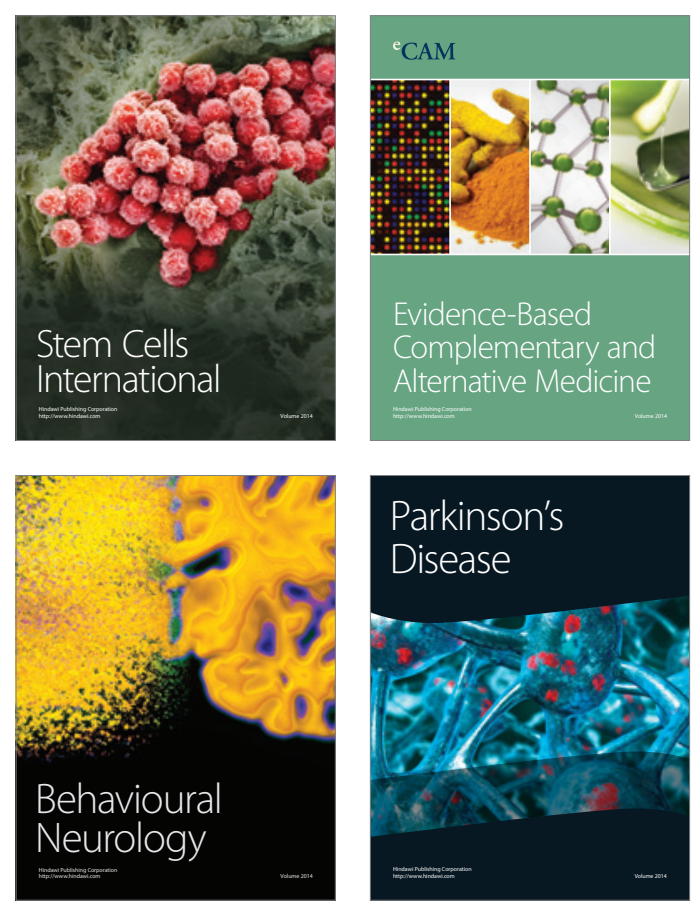

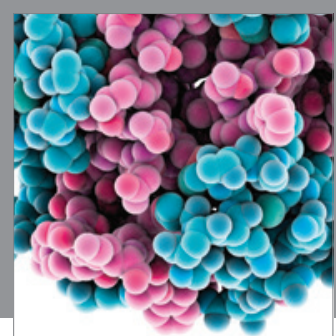

Journal of
Diabetes Research

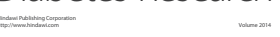

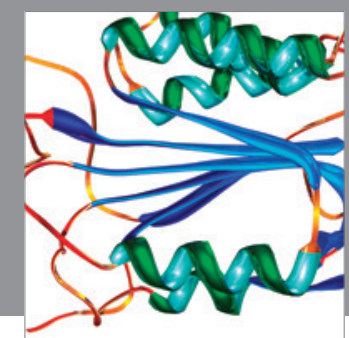

Disease Markers
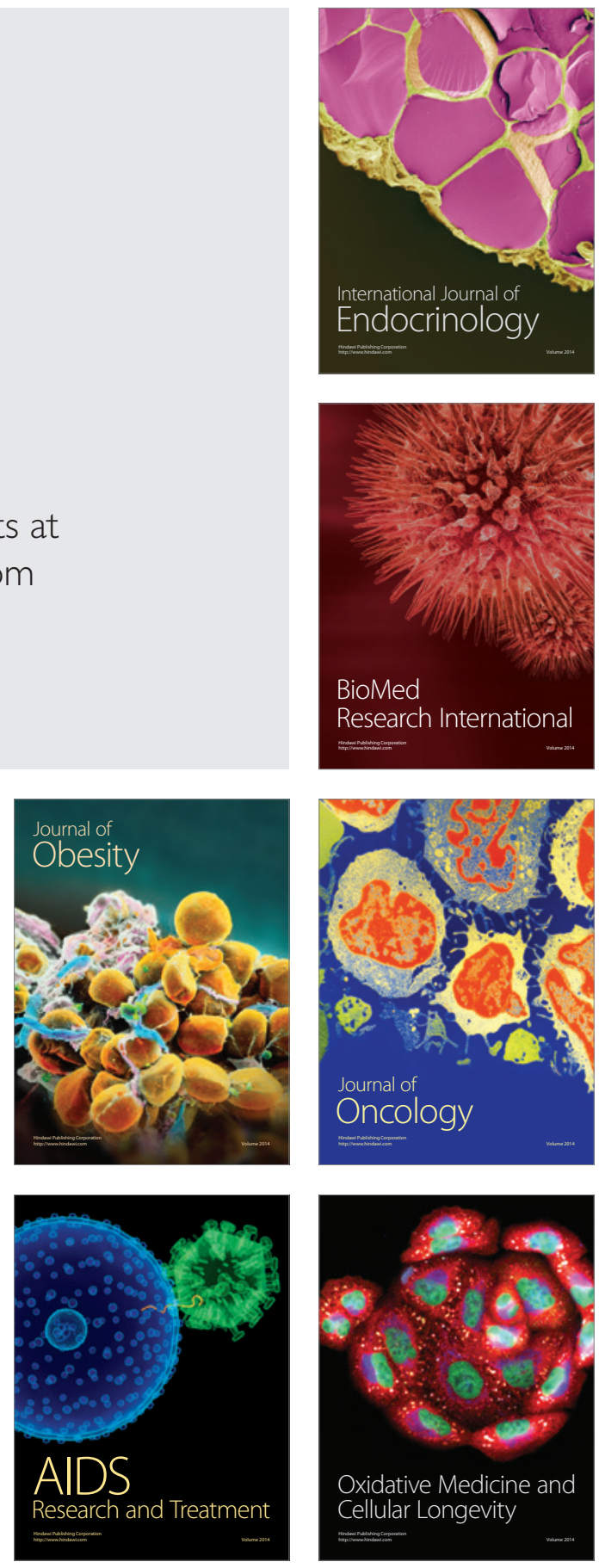